\title{
Concepciones sobre enseñanza y aprendizaje de las Ciencias Naturales de educadoras de párvulos en formación en Chile y sus relaciones con modelos de racionalidad científica
}

\author{
Conceptions of teaching and learning Natural Sciences \\ in early childhood educators in Chile and their relationships \\ with models of scientific rationality
}

\author{
Carolina Orellana-Sepúlveda ${ }^{1}$ https://orcid.org/0000-0002-4291-1705 \\ Mario Roberto Quintanilla-Gatica ${ }^{1}$. https://orcid.org/0000-0002-4411-7919 \\ Rodrigo Páez-Cornejo ${ }^{1}$. https://orcid.org/0000-0002-6615-5721
}

\begin{abstract}
Resumen: Desde un posicionamiento praxeológico, las concepciones sobre enseñanza y aprendizaje cobran un rol importante en la puesta en práctica del currículum prescrito y en la configuración de las experiencias de aprendizaje en el aula. Este trabajo tiene por objetivo identificar y caracterizar concepciones sobre enseñanza y aprendizaje de las ciencias naturales en Educadoras de Párvulos en formación al inicio de un curso de Didáctica de las Ciencias Naturales. Los resultados indican que existe una coexistencia entre posiciones cercanas a modelos con rasgos de un racionalismo fuerte, radical, y nociones sobre la enseñanza y el aprendizaje de las ciencias de tipo tradicional-dogmática con características de un racionalismo moderado y una noción constructivista respecto al aprendizaje.
\end{abstract}

Palabras clave: Educadoras de párvulos. Enseñanza y aprendizaje. Enseñanza de las ciencias. Pensamiento científico.

\begin{abstract}
From a praxeological position, conceptions about teaching and learning take an important role in the implementation of the prescribed curriculum and in the configuration of learning experiences in the classroom. The objective of this work is to identify and characterize conceptions about teaching and learning of the natural sciences in early childhood educators in training at the beginning of a course in the Didactics of Natural Sciences. The results indicate that there is a coexistence between positions close to models with traits having a strong, radical rationalism, and notions about the teaching and learning of traditional-dogmatic-type sciences with characteristics of a moderate rationalism and a constructivist notion with respect to learning.
\end{abstract}

Keywords: Early childhood teaching. Teaching and learning. Science education. Scientific thinking.

\footnotetext{
${ }^{1}$ Pontificia Universidad Católica de Chile, Facultad de Educación, Santiago, Chile. E-mail: <corellanas@uc.cl>.
} 


\section{Introducción}

Desde una perspectiva interdisciplinaria, este trabajo emana de un proyecto que se propone identificar, caracterizar y promover competencias de pensamiento científico (CPC) en la formación inicial de Educadoras de Párvulos, contribuyendo a la profesionalización docente para la primera infancia y al desarrollo de la alfabetización científica con calidad y con equidad. Esta investigación es la continuación de una serie de proyectos de investigación y desarrollo (I \& D) de carácter nacional y de cooperación internacional iniciados hace ya una década en el Laboratorio de Investigación en Enseñanza de las Ciencias de la Pontificia Universidad Católica de Chile (GRECIA-UC) ${ }^{2}$. Este nuevo proyecto viene a ampliar y profundizar las innumerables acciones de investigación, divulgación, gestión y formación ya desarrolladas para develar las concepciones teóricas sobre las Ciencias Naturales y su enseñanza, evaluación y aprendizaje, en el campo profesional docente.

Parece claro que hoy existen pocos referentes objetivos para vincular el desarrollo de CPC con la enseñanza efectiva de las Ciencias Naturales en la Educación Parvularia. En esta comunicación sólo adelantamos una parte de la investigación con su componente epistemológica y metodológica. Se trata de identificar y caracterizar las concepciones sobre enseñanza y aprendizaje de las ciencias naturales de Educadoras de Párvulos en Formación al Inicio de un Curso de Didáctica de las Ciencias Naturales.

Aprender a resolver problemas específicos y a responder a las necesidades de la sociedad utilizando el conocimiento y las competencias de pensamiento científico y otras habilidades científicas y tecnológicas es hoy parte de esa educación que queremos para nuestros estudiantes (PRINGLE, 2004). La formación de los educadores que deberán emprender esta tarea de enseñanza en todos los niveles educativos, resulta un factor crítico para el éxito. En efecto, estudios internacionales demuestran que existe una alta correlación entre la calidad de las competencias docentes y los logros de aprendizajes de sus estudiantes (BENNET, 2010; MONTECINOS et al., 2009). Para el contexto chileno, el Informe sobre desarrollo humano 2009 (INFORME..., 2009) muestra como el desafío de aumentar las oportunidades del país y de traducirlas en resultados concretos para las personas, se relaciona precisamente con la transformación de sus competencias; es decir, con los modos de actuación y de relación que las personas despliegan en espacios concretos de acción educativa formal, no formal e informal. No obstante este consenso sobre el desarrollo de competencias de pensamiento científico (CPC) y aprendizajes de los niños en educación parvularia (EP) en Chile, no cuenta con un abundante reporte de su situación (FALABELLA; ROJAS, 2008). Visto así, la formación inicial docente y el desarrollo de estas competencias científicas, se torna un imperativo tanto pedagógico-científico como ético-político justificando su investigación. En concreto, una de las preocupaciones de la ciencia es comprender el mundo natural e intervenir en los cambios que la actividad humana produce y, en función del conocimiento que inventa y construye, tomar decisiones sobre cómo actuar. Para conseguir estas finalidades busca identificar preguntas relevantes, generar conceptos, modelos y teorías para darles respuesta y encontrar pruebas que las confirmen o den lugar a nuevas preguntas (MERINO; SANMARTÍ, 2008).

\footnotetext{
${ }^{2}$ Información disponible en: < https://laboratoriogrecia.cl/>.
} 


\section{Desarrollo de la investigación y orientaciones teóricas}

Desde un posicionamiento praxeológico, el currículum será interpretado, enriquecido o empobrecido. Será transformado por prácticas en instancias políticas, administrativas, por los materiales curriculares, por los centros escolares y por el profesor. (GIMENO, 2010). Bajo este supuesto, el currículum oficial o prescrito es interpretado por el docente, convirtiéndose éste en un mediador que toma decisiones influenciado por su cultura, saberes y concepciones (MARRERO, 2010). Sanmartí (2002) afirma que la investigación muestra que el profesorado siempre transforma el currículo, y aquello que enseña tiene más que ver con sus concepciones sobre la ciencia y las finalidades de su enseñanza que con las orientaciones oficiales o los conocimientos didácticos. Desde esta visión práctica del currículum, la Educadora de Párvulos se concibe como mediadora en la construcción de los aprendizajes del niño y responsable de convertir las propuestas del currículum en actividades de aprendizaje que respondan a un "deber ser" (RÍOS; ANGULO, 2011) de la enseñanza de las ciencias propuestas en el currículum. La valoración y toma de decisiones que realiza el docente sobre un contenido y el énfasis que pone en su enseñanza es reflejo de su propia cultura, sus ponderaciones personales, sus actitudes hacia la enseñanza de ciertas áreas, sus concepciones implícitas acerca de la enseñanza (MARRERO, 2010; SANMARTÍ, 2002), sin embargo, las decisiones que toman las Educadoras sobre el currículum no deben ser producto del azar o de prácticas reproductivas, por lo que el profesorado que enseña ciencias, en este caso la educadora enfrentada a esta tarea, debe disponer de criterios fundamentados en referentes teóricos y prácticos (SANMARTÍ, 2002) por lo que se hace indispensable que la formación inicial docente se haga cargo de esta necesidad formando profesores que reflexionan sobre sus representaciones de la ciencia, su naturaleza, enseñanza y aprendizaje en la primera infancia.

Desde la década de 1980 la investigación sobre las concepciones docentes bajo diversas perspectivas se ha convertido en una línea de trabajo prioritaria (PORLÁN; MARTÍN DEL POZO, 2004). Las concepciones de los docentes respecto de la enseñanza y aprendizaje de las ciencias han sido abordadas sobre todo a nivel de enseñanza secundaria y primaria, sin embargo, sabemos poco sobre las concepciones de docentes que imparten ciencias en las primeras edades y menos aún, en Educadores(as) de Párvulos en formación en Chile. Carvajal y Gómez (2002), en un estudio relacionado a las concepciones sobre la naturaleza y aprendizaje de las ciencias, plantean que los docentes no son conscientes de sus concepciones y que, estas permanecen estables a pesar de la formación docente.

El término concepción ha sido polémico en el campo de la investigación debido a la imprecisión con la que se ha usado el mismo, por ejemplo, en algunos artículos los autores se refieren a concepciones y luego pasan a utilizar indistintamente el concepto de creencia. Fernández et al. (2011) profundizan en esta diversidad terminológica para referirse a las concepciones docentes y precisan, citando a sus respectivos autores, que se han utilizado términos como creencias, teorías implícitas, filosofías pedagógicas y conocimiento práctico.

La concepción de ciencia, su naturaleza, finalidades, métodos y construcción de productos de conocimiento, influyen u orientan acciones e ideas sobre el ejercicio profesional del profesorado (QUINTANILLA, 2006), estableciéndose vinculaciones con modelos didácticos de enseñanza y también de aprendizaje, formas de considerar y valorar el acto de aprender, el desarrollo de los sujetos que aprenden, diseños que organizan contenidos, actividades para el 
aprendizaje y sus formas de evaluación (estrategias e instrumentos) (ALIBERAS; GUTIERREZ; IZQUIERDO, 1989; QUINTANILLA, 1999).

El profesorado en sus acciones educativas transfiere y modela, con su lógica y visión propia, basándose en sus experiencias tanto de desarrollo profesional como extra-profesional, modelos de racionalidad científica en cuanto a las prácticas y formas de desarrollar conocimiento. Dentro de las formas de ver y pensar la ciencia se reconocen dos modelos de racionalismo, por una parte, un racionalismo radical o fuerte (RR) que no admite la valoración del juicio científico en la toma de decisiones y que explica la realidad de forma perfecta y categórica, por lo tanto dice la verdad (QUINTANILLA, 2006). En este modelo, las relaciones entre teoría y dato son exclusivamente racionales (GIERE, 1990), constituyendo el criterio de racionalidad un requisito para la distinción entre ciencia y no ciencia (QUINTANILLA, 1999; SCHLICK, 1965), sin considerarse los contextos en los que se desarrolla la actividad científica, ya sea personal, social o cultural, estos elementos se consideran irrelevantes para una adecuada comprensión de y sobre la ciencia (GIERE, 1992). Este tipo de racionalidad se vincula con modelos de enseñanza de tipo tradicional-dogmático, donde la tendencia es a la transmisión de saberes inamovibles por parte de quien enseña y de recepción en el caso de quien estaría aprendiendo. Por otra parte, en un modelo de racionalismo moderado (RM) las teorías se aceptan o se rechazan a través de un proceso de validación que involucra el juicio individual del científico y la interacción social de los mismos (GIERE, 1992), lo que va modelando la construcción y selección de las nuevas teorías y explicaciones respecto a un fenómeno estudiado, y en base a estas explicaciones se resuelven problemas. Por lo tanto, en este modelo no solo se considera el acto racional dentro de la complejidad de la construcción de las nuevas teorías, sino también aquellas condiciones que influyen o inducen a los científicos en la forma de desarrollar y justificar los descubrimientos científicos. Este tipo de racionalidad se aproxima a un modelo didáctico de construcción o socio-constructivista, donde el aprendizaje se construye en base a las subjetividades, concepciones y teorías personales de los sujetos que aprenden, interactuando en un contexto particular.

\section{Materiales y Métodos}

Este trabajo tiene como objetivos identificar y caracterizar las concepciones sobre enseñanza y aprendizaje de las ciencias naturales de Educadoras de Párvulos en formación al inicio de un curso de Didáctica de las Ciencias Naturales (DCN) y corresponde a los primeros hallazgos para caracterizar las transiciones de pensamiento cientifico que sigue un grupo de Educadoras de Párvulos en formación al participar en grupos de discusión (Talleres de Reflexión Docente o TRD) en torno a la enseñanza y aprendizaje de las ciencias para promover competencias de pensamiento científico (CPC), en el marco de una intervención intencionada en el plan curricular del curso DCN y como parte de la segunda fase del Proyecto FONDECYT 1150505: Identificación y caracterización de las competencias de pensamiento cientifico de educadoras de párvulos en formación: una contribución fundamental para la educación cientifica en las primeras edades que dirige el segundo autor. Los resultados presentados corresponden al punto de inflexión que marca el inicio de estas transiciones de pensamiento a partir del análisis de un cuestionario aplicado al principio del curso. La intervención curricular en el plan del curso se traduce en la introducción 'intencionada' de 6 Talleres de Reflexión docente (TRD) en temáticas de: Concepciones sobre Enseñanza y 
Aprendizaje de las ciencias, Rol del docente de ciencias y diseño de Experiencias de Aprendizaje para párvulos, Naturaleza de la Ciencia, Competencias de Pensamiento Científico en Educación de Párvulos, Resolución de Problemas Científicos y Evaluación de aprendizajes científicos.

Al comienzo del curso se ha aplicado un cuestionario estilo Likert que tiene la finalidad de caracterizar las ideas y valoraciones iniciales que las profesionales en formación tienen sobre la enseñanza de las ciencias naturales en la primera infancia. El instrumento se organiza en 7 dimensiones: Naturaleza de la Ciencia, Enseñanza de las Ciencias, Aprendizaje de las Ciencias, Evaluación de los Aprendizajes Científicos, Rol de los educadores de Ciencias Naturales, Resolución de Problemas Científicos, y Competencias de Pensamiento Científico. Cada dimensión consta de diez enunciados (a excepción de la dimensión 7 que tiene nueve) en donde se les solicita emitir su grado de acuerdo con cada afirmación según la escala de valoración presentada en la Tabla 1. Para efectos del análisis de resultados a cada valoración se le ha asignado un valor especificado en la misma tabla.

Tabla 1. Escala de valoración de afirmaciones, clave y valor asignado

\begin{tabular}{lcc}
\hline \multicolumn{1}{c}{ Valoraciones } & Clave & Valor asignado \\
\hline Totalmente de acuerdo & TA & 4 \\
Parcialmente de acuerdo & PA & 3 \\
Parcialmente en desacuerdo & PD & 2 \\
Totalmente en desacuerdo & TD & 1 \\
\hline
\end{tabular}

Fuente: Elaboración de los autores.

En los Cuadros 1 y 2 se definen las afirmaciones para la Dimensión 2 y 3 correspondientes a Enseñanza de las Ciencias y Aprendizaje de las Ciencias respectivamente.

Cuadro 1. Dimensión 2: Enseñanza de las Ciencias (EDC)

\begin{tabular}{|l|l|}
\hline Código & \multicolumn{1}{|c|}{ Afirmación } \\
\hline EDC1 & $\begin{array}{l}\text { La ciencia que se enseña en el aula es un conocimiento que no incluye componentes } \\
\text { ideológicos, sociales y culturales. }\end{array}$ \\
\hline EDC2 & $\begin{array}{l}\text { La enseñanza de las ciencias naturales permite que los párvulos remplacen sus modelos } \\
\text { incorrectos acerca de la realidad, por concepto científicamente correctos. }\end{array}$ \\
\hline EDC3 & $\begin{array}{l}\text { Las actividades experimentales son imprescindibles para justificar la enseñanza de teorías } \\
\text { científicas. }\end{array}$ \\
\hline EDC4 & $\begin{array}{l}\text { La enseñanza de las ciencias naturales en el aula debe considerar el significado que los } \\
\text { párvulos tienen de un concepto, aunque este no corresponda con el significado correcto. }\end{array}$ \\
\hline EDC5 & $\begin{array}{l}\text { La enseñanza de teorías científicas debe promover la relación entre los conceptos } \\
\text { científicos, en los diferentes campos de un saber erudito (Culto/ Sabio) }\end{array}$ \\
\hline EDC6 & $\begin{array}{l}\text { La enseñanza de las ciencias naturales promueve en los párvulos una actitud ciudadana } \\
\text { crítica y responsable. }\end{array}$ \\
\hline EDC7 & $\begin{array}{l}\text { La enseñanza reflexiva del método científico permite que los párvulos cambien su forma } \\
\text { de actuar frente a nuevas situaciones del mundo real }\end{array}$ \\
\hline
\end{tabular}


Cuadro 1. continuación

\begin{tabular}{|l|l|}
\hline Código & \multicolumn{1}{|c|}{ Afirmación } \\
\hline EDC8 & $\begin{array}{l}\text { La enseñanza de las ciencias naturales permite explicar el mundo cotidiano con teorías } \\
\text { científicas. }\end{array}$ \\
\hline EDC9 & $\begin{array}{l}\text { En la enseñanza de las ciencias naturales se obtienen aprendizajes definitivos, aún si no se } \\
\text { consideran los conocimientos previos. }\end{array}$ \\
\hline EDC10 & $\begin{array}{l}\text { La enseñanza de las ciencias naturales se basa en dejar que los párvulos descubran, por sí } \\
\text { mismos, los conceptos científicos. }\end{array}$ \\
\hline
\end{tabular}

Fuente: Quintanilla (2015).

Cuadro 2. Dimensión 3: Aprendizaje de las Ciencias (ADC)

\begin{tabular}{|l|l|}
\hline Código & \multicolumn{1}{|c|}{ Afirmación } \\
\hline ADC1 & $\begin{array}{l}\text { El aprendizaje de las ciencias naturales se adquiere en un proceso colectivo, por el cual } \\
\text { los párvulos elaboran conocimientos que pueden o no coincidir con las teorías de los } \\
\text { científicos. }\end{array}$ \\
\hline ADC2 & $\begin{array}{l}\text { Aprender a aprender ciencias naturales, implica evaluar y co-evaluar con los pares, las } \\
\text { distintas actividades que promueven los educadores. }\end{array}$ \\
\hline ADC3 & $\begin{array}{l}\text { Las teorías científicas que se aprenden en las experiencias de ciencias naturales, tienen } \\
\text { relación directa con los modelos científicos válidamente aceptados. }\end{array}$ \\
\hline ADC4 & $\begin{array}{l}\text { El aprendizaje científico de la escuela y el jardín infantil es un proceso por el cual los } \\
\text { párvulos relacionan su conocimiento, tanto con el de sus pares como el de otras fuentes } \\
\text { de recursos. }\end{array}$ \\
\hline ADC5 & $\begin{array}{l}\text { El aprendizaje científico de la escuela y el jardín infantil, se produce cuando los } \\
\text { educadores reemplazan las concepciones incorrectas de los párvulos por las de las teorías } \\
\text { científicas. }\end{array}$ \\
\hline ADC6 & $\begin{array}{l}\text { Las teorías con las cuales los párvulos interpretan el mundo cambian después de un } \\
\text { proceso de aprendizaje de las ciencias naturales. }\end{array}$ \\
\hline ADC7 & $\begin{array}{l}\text { El aprendizaje científico de la escuela y el jardín infantil, permite que el párvulo sustituya } \\
\text { totalmente las ideas previas o cotidianas poco elaboradas, por otras del ámbito científico. }\end{array}$ \\
\hline ADC8 & $\begin{array}{l}\text { Los párvulos deben participar en las decisiones acerca de qué y cómo aprender, porque } \\
\text { ellos son responsables de su aprendizaje científico. }\end{array}$ \\
\hline ADC9 & $\begin{array}{l}\text { Los párvulos pueden aprender activamente conceptos científicos inapropiados fuera de } \\
\text { la escuela para interpretar la realidad y su propia experiencia. }\end{array}$ \\
\hline ADC10 & $\begin{array}{l}\text { En el aprendizaje de las ciencias naturales cada educador proporciona a los párvulos } \\
\text { información necesaria, para que estos organicen su propia experiencia. }\end{array}$ \\
\hline
\end{tabular}

Fuente: Quintanilla (2015).

Las categorías establecidas para el análisis de los datos se establecen a partir de la literatura (IZQUIERDO et al., 1999; QUINTANILLA, 1999, 2006; RAVANAL; QUINTANILLA, 2012; SANMARTÍ; GARCÍA, 1999) estas categorías responden a la asociación teórica que se ha realizado entre visiones de ciencia asociadas a los modelos de racionalidad y enfoques de enseñanza. Así, por un lado se ha asociado el Racionalismo Radical con una Noción Tradicio- 
nal-Dogmática de enseñanza y aprendizaje, por otro, se asocia el Racionalismo Moderado con una noción constructivista de la enseñanza y el aprendizaje. La clasificación de cada una de las aseveraciones del cuestionario según estas categorías de análisis se presenta en el Cuadro 3.

Cuadro 3. Asociación de afirmaciones de cada dimensión con las categorías de análisis propuestas

\begin{tabular}{|l|c|c|c|}
\hline & $\begin{array}{c}\text { Racionalismo Radical y } \\
\text { noción Tradicional-Dogmática }\end{array}$ & $\begin{array}{c}\text { Racionalismo Moderado y noción } \\
\text { Constructivista }\end{array}$ \\
\hline D2: Enseñanza de las & EDC1 & \multicolumn{2}{|c|}{ EDC4 } \\
Ciencias (EDC) & EDC2 & \multicolumn{2}{|c|}{ EDC5 } \\
& EDC6 \\
& EDC3 & \multicolumn{2}{|c|}{ EDC7 } \\
\hline EDC9 & ADC1 & ADC8 \\
ED3: Aprendizaje de las & ADC3 & ADC2 & ADC9 \\
Ciencias (ADC) & ADC5 & ADC4 & ADC10 \\
& ADC7 & ADC6 & \\
\hline
\end{tabular}

Fuente: elaboración de los autores.

El análisis de resultados y la construcción de gráficos se realizó mediante el software de análisis estadístico IBM SPSS Statistics Versión 23.0.0.0 (IBM..., 2016). La muestra está compuesta por 26 estudiantes de Educación Parvularia (infantil) que cursan la asignatura Pensamiento Científico del Niño y su Didáctica durante el segundo semestre de 2016.

\section{Resultados}

\section{Dimensión 2: enseñanza de las ciencias (EDC)}

Tabla 2. Estadísticos descriptivos dimensión 2: Enseñanza de las ciencias

\begin{tabular}{lcccccccccc}
\hline & EDC1 & EDC2 & EDC3 & EDC4 & EDC5 & EDC6 & EDC7 & EDC8 & EDC9 & EDC10 \\
\hline $\mathrm{N}$ Válido & 26 & 26 & 26 & 26 & 25 & 26 & 26 & 26 & 26 & 26 \\
$\quad$ Perdidos & 0 & 0 & 0 & 0 & 1 & 0 & 0 & 0 & 0 & 0 \\
Media & 2,46 & 2,85 & 3,69 & 3,96 & 3,56 & 3,85 & 3,65 & 3,69 & 2,12 & 3,19 \\
Moda & 3 & 3 & 4 & 4 & 4 & 4 & 4 & 4 & 3 & 4 \\
Desviación & 1,029 & 0,881 & 0,679 & 0,196 & 0,507 & 0,464 & 0,485 & 0,618 & 0,909 & 0,849 \\
estándar & & & & & & & & & \\
Varianza & 1,058 & 0,775 & 0,462 & 0,038 & 0,257 & 0,215 & 0,235 & 0,382 & 0,826 & 0,722 \\
\hline
\end{tabular}

Fuente: elaboración de los autores. 
Respecto a las afirmaciones relacionadas a concepciones epistemológicas que tienen como base un Racionalismo Moderado y que se asocian a nociones constructivistas los estadísticos descriptivos, muestran que existe un acuerdo parcial con aquellas afirmaciones, el 100\% de ellas presentan una moda de 4 correspondiendo a la valoración Totalmente de acuerdo y con medias cercanas a 4 .

Sobre las afirmaciones relacionadas epistemológicamente a un Racionalismo Radical y una noción de enseñanza Tradicional-Dogmática de las ciencias encontramos que existe un acuerdo general con aquellas afirmaciones, predominando un grado de acuerdo relacionado a la preferencia "Parcialmente de acuerdo" a excepción de EDC3 "Las actividades experimentales son imprescindibles para justificar la enseñanza de teorías científicas" que presenta una media de 3.69 y una moda de 4, correspondiente a la preferencia Totalmente de Acuerdo.

Es de interés destacar que todas las aseveraciones de esta categoría presentan una desviación estándar superior a 0,6 lo que da cuenta de una alta dispersión en las respuestas. Se espera profundizar en el tránsito de la preferencia EDC1 "La ciencia que se enseña en el aula es un conocimiento que no incluye componentes ideológicos, sociales y culturales" que presenta una desviación estándar alta $(1,029)$ lo que representa una alta dispersión de las preferencias por lo que es difícil establecer que existe un grado de acuerdo frente a la afirmación (Gráfico 1).

Gráfico 1. Preferencias sobre la afirmación EDC1

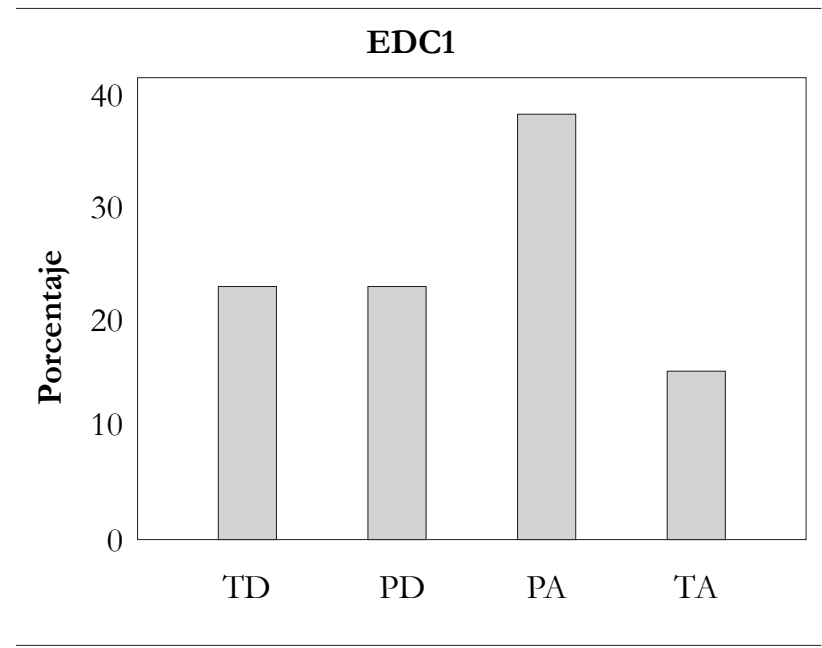

Fuente: elaboración de los autores. 


\section{Dimensión 3: aprendizaje de las ciencias (ADC)}

Tabla 3. Estadísticos descriptivos Dimensión 3: Aprendizaje de las ciencias

\begin{tabular}{lcccccccccc}
\hline & ADC1 & ADC2 & ADC3 & ADC4 & ADC5 & ADC6 & ADC7 & ADC8 & ADC9 & ADC10 \\
\hline N Válido & 26 & 26 & 24 & 26 & 25 & 25 & 25 & 25 & 25 & 25 \\
$\quad$ Perdidos & 0 & 0 & 2 & 0 & 1 & 1 & 1 & 1 & 1 & 1 \\
Media & 3,50 & 3,69 & 3,21 & 3,92 & 2,32 & 3,08 & 2,52 & 3,56 & 3,36 & 3,28 \\
Moda & 4 & 4 & 3 & 4 & 2 & 3 & 3 & 4 & 4 & 3 \\
Desviación & 0,707 & 0,549 & 0,588 & 0,272 & 0,900 & 0,812 & 1,085 & 0,651 & 0,810 & 0,678 \\
estándar & & & & & & & & & & \\
Varianza & 0,500 & 0,302 & 0,346 & 0,074 & 0,810 & 0,660 & 1,177 & 0,423 & 0,657 & 0,460 \\
\hline
\end{tabular}

Fuente: elaboración de los autores.

Respecto de las afirmaciones asociadas con concepciones epistemológicas de Racionalismo moderado y concepciones de aprendizaje constructivistas existe un acuerdo generalizado del grupo frente a las afirmaciones con preferencias que van entre totalmente de acuerdo y parcialmente de acuerdo.

El grupo de educadoras se presenta parcialmente de acuerdo con dos de las tres afirmaciones relacionadas con una noción del aprendizaje ligado a un Dogmatismo Positivista y una concepción de aprendizaje Tradicional-Dogmática. Para ADC3 "Las teorías científicas que se aprenden en las experiencias de ciencias naturales, tienen relación directa con los modelos científicos válidamente aceptados" se presenta una media $(3,21)$ y una moda (3) coincidente con una preferencia de "parcialmente de acuerdo" frente a la afirmación. Llama la atención que para ADC7 "El aprendizaje cientifico de la escuela y el jardín infantil, permite que el párvulo sustituya totalmente las ideas previas o cotidianas poco elaboradas, por otras del ámbito científico" también la moda se atribuye a la preferencia "parcialmente de acuerdo", sin embargo, llama la atención la alta dispersión de datos de la afirmación (Gráfico 2).

Gráfico 2. Preferencias sobre la afirmación ADC7

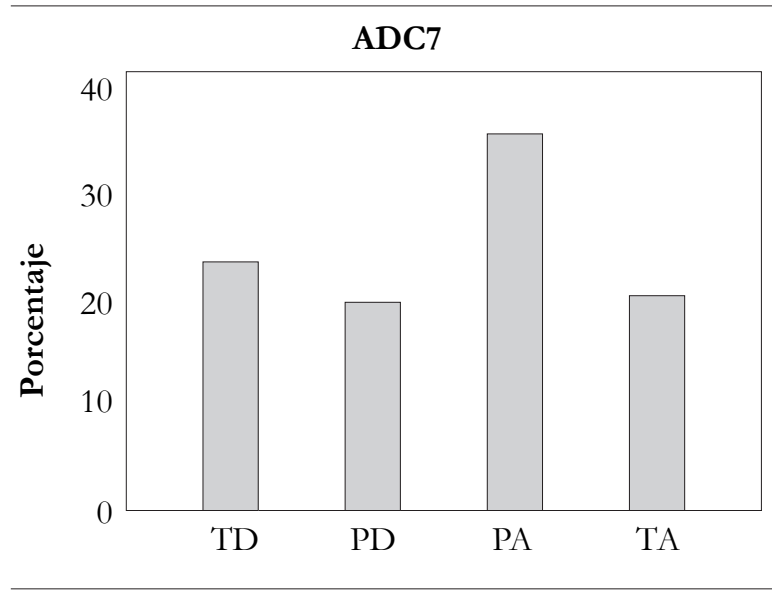

Fuente: elaboración de los autores. 
Finalmente, también resultará interesante profundizar en la afirmación ADC5 "El aprendizaje cientifico de la escuela y el jardin infantil, se produce cuando los educadores reemplazan las concepciones incorrectas de los párvulos por las de las teorías científicas" ya que también presenta una alta dispersión en las preferencias.

\section{Conclusiones y proyecciones del estudio}

Preliminarmente, se identifica que las educadoras en formación presentan concepciones sobre enseñanza y aprendizaje que se pueden asociar tanto a un Racionalismo Radical y una Noción Tradicional-Dogmática de la enseñanza y el aprendizaje de las Ciencias, como a un Racionalismo Moderado y una noción constructivista del mismo.

De esta forma, se observa una coexistencia entre concepciones que podríamos calificar como contradictorias, situación se evidencia en otros estudios relacionados a concepciones y creencias de los docentes en relación a la enseñanza y aprendizaje de las Ciencias, en donde manifiestan a menudo posiciones relacionadas con una concepción transmisiva de la enseñanza y concepciones de aprendizaje por incorporación de significados externos, entre otras características, lo que es coherente con la cultura tradicional en la que los futuros profesionales se han formado (PORLÁN et al., 2010).

En un contexto donde surgen propuestas curriculares asociadas a elementos de enfoques basados en la construcción social del conocimiento (SANMARTÍ; GARCÍA, 1999) resulta problemático que persistan ideas de tipo Racionalista Radical en los albores del siglo XXI donde las finalidades de la enseñanza de las ciencias han cambiado (SANMARTÍ, 2002) hacia el logro de una alfabetización científica para la ciudadanía que permita una comprensión de la ciencia para favorecer la toma de decisiones responsables (MARCO-STIEFEL, 2000) respecto a los efectos globales del actual desarrollo socioeconómico y el uso de los recursos planetarios, que beneficia a un fragmento reducido de la población (FURIÓ et al., 2001).

Ya desde hace unos cuantos años se discute que la formación actual del profesorado no responde a las finalidades actuales de la enseñanza de las ciencias (SANMARTÍ, 2002). Quintanilla (1999) plantea que en las instituciones formadoras de profesores aún coexisten modelos de ciencia y enseñanza de las ciencias que no han superado la visión positivista del siglo XIX lo que se traduce en clases de ciencias tradicionales en distintos niveles de formación, con predominio de un modelo didáctico de transmisión recepción (LABARRERE; QUINTANILLA, 2006). En este contexto, consideramos que estudiar las transiciones de pensamiento a partir de la participación de Educadoras de Párvulos en formación en Talleres de Reflexión Docente, donde se posicionan como profesionales que reflexionan y toman conciencia acerca de estos temas, constituye un aporte al campo de investigación y a la formación de educadoras que deberán hacerse cargo de la compleja tarea de enseñar ciencias en la primera infancia. En este sentido, cabe destacar que la relevancia de este proyecto está tanto en el aporte que hace desde el componente investigativo, como desde su componente de desarrollo tecnológico. Por un lado, se construye conocimiento sobre la formación inicial docente de EP, con foco en la enseñanza y aprendizaje de las ciencias en general y en la promoción de competencias de pensamiento científico en particular; un cruce de campos de estudio, que para la Educación Parvularia (infantil) se encuentran en progresiva emergencia y valorización. 
Por otro lado, el proyecto se constituye en el pilotaje de un auténtico proceso de profesionalización para este nivel en el área de las ciencias. Esto, toda vez que prueba con profesionales del nivel una metodología que les permite acceder de manera consciente a los procesos, condiciones y productos que tienen lugar durante la formación de CPC, e inscribirlos en una corriente sistemática de juicios valorativos y evaluativos. Nos proponemos así desentrañar, en el marco de esta complejidad, los entendimientos intra e interpersonales sobre las competencias científicas, desde las cuales las EP experimentan y dan sentido a su actuar pedagógico-formativo.

\section{Agradecimientos}

Los autores de este trabajo agradecen a los proyectos FONDECYT 1150505, REDES 150107 y a la Comisión Nacional Científica y Tecnológica de Chile (CONICYT).

\section{Referencias}

ALIBERAS, J.; GUTIERREZ, R.; IZQUIERDO, M. Modelos de aprendizaje en la didáctica de las ciencias. Investigación en la Escuela, Sevilla, n. 9, p. 17-24, 1989. Recuperado de: <http:/ /hdl.handle.net/11441/59223>. Accedido el: 10 out. 2018.

BENNET, B. The artful science of instructional integration. In: MARZANO, R. (Ed.). On excellence in teaching. Bloomington: Solution Tree Press, 2010. p. 65-91.

CARVAJAL E.; GÓMEZ M. Concepciones y representaciones de los maestros de secundaria y bachillerato sobre la naturaleza, el aprendizaje y la enseñanza de las ciencias. Revista Mexicana de Investigación Educativa, Mexico, v. 7, n. 16, p. 557-602, 2002. Recuperado de: <http://www.redalyc.org/articulo.oa?id=14001607>. Accedido el: 11 out. 2018.

FALABELLA, A.; ROJAS, M. Algunas tendencias curriculares en la formación de educadores de párvulos. Calidad en la Educación, Santiago, n. 29, p. 159-191, 2008.

FERNÁNDEZ, M. et al. Concepciones sobre la enseñanza del profesorado y sus actuaciones en clases de ciencias naturales de educación secundaria. Revista Mexicana de Investigación Educativa, Mexico, v. 16, n. 49, p. 571-596, 2011. Recuperado de: <http:// www.scielo.org.mx/pdf/rmie/v16n49/v16n49a11.pdf>. Accedido el: 4 ene. 2018.

FURIÓ, C. et al. Finalidades de la enseñanza de las ciencias en la secundaria obligatoria: ¿alfabetización científica o preparación propedéutica? Enseñanza de las Ciencias, Barcelona, v. 19, n. 3, p. 365-376, 2001. Recuperado de: <https://ddd.uab.cat/record/1538>. Accedido el: 4 ene. 2018.

GIERE, R. Explaining science: a cognitive approach. Chicago: The University of Chicago Press, 1990.

GIERE, R. (Ed). Cognitive models of science. Minneapolis: University of Minnesota Press, 1992. 
GIMENO, J. ¿Qué significa el currículum? In: GIMENO, J. Saberes e incertidumbres sobre el currículum. Madrid: Morata, 2010. p. 21-43.

IBM SPSS Statistics: version 23.0. 2016. Recuperado de: <https://www-01.ibm.com/ support/docview.wss?uid =swg24040559>. Accedido el: 10 out. 2018.

INFORME sobre desarrollo humano 2009: superando barreras: movilidad e desarrollo humanos. Madrid: PNUD, 2009. Recuperado de: <http://desarrollohumano.cl/idh/ download/hdr_2009_es_complete.pdf>. Accedido el: 9 oct. 2018.

IZQUIERDO, M. et al. Caracterización y fundamentación de la ciencia escolar. Enseñanza de las Ciencias, Barcelona, v. 17, n. 1, p. 45-59, 1999.

LABARRERE, A.; QUINTANILLA, M. La evaluación de los profesores de ciencia desde la profesionalidad emergente. In: QUINTANILLA, M.; ADÚRIZ-BRAVO, A. (Ed.). Enseñar ciencias en el nuevo milenio: retos y propuestas. Santiago: Universidad Católica de Chile, 2006. p. 257-278.

MARCO-STIEFEL, B. La alfabetización científica. In: PERALES, F.; CAÑAL, P. (Ed.). Didáctica de las ciencias experimentales. Alcoy: Marfil, 2000. p. 141-186.

MARRERO, J. El currículum que es interpretado: ¿que enseñan los centros y los profesores y profesoras? In: GIMENO, J. (Comp.). Saberes e incertidumbres sobre el currículum. Madrid: Morata, 2010. p. 221-245.

MERINO, C.; SANMARTÍ, N. How young children model chemical change. Chemistry Education Research and Practice, Ioannina, v. 9, p. 196-207, 2008. Recuperado de: <https://doi.org/10.1039/B812408F>. Accedido el: 9 oct. 2018.

MONTECINOS, C. et al. Muestras de desempeño docente: un instrumento para evaluar la calidad de la enseñanza y su impacto en el aprendizaje. Santiago de Chile: Ediciones Universidad Católica de Chile, 2009.

PRINGLE, R. M. Scholarship in science education. Journal of Elementary Science

Education, Dordrecht, v. 16, n. 1, p. 9-19, 2004. Recuperado de: <https://doi.org/10.1007/ BF03174747>. Accedido el: 9 oct. 2018.

PORLÁN, R. et al. El cambio del profesorado de ciencias I: marco teórico y formativo.

Enseñanza de las Ciencias, Barcelona, v. 28, n. 1, p. 31-46, 2010. Recuperado de:

< https://www.raco.cat/index.php/Ensenanza/article/view/189094/353373>. Accedido el: 9 oct. 2018.

PORLÁN, R.; MARTÍN DEL POZO, R. The conceptions of in-service and prospective primary school teachers about the teaching and learning of science. Journal of Science Teacher Education, Abingdon, v. 15, n. 1, p. 39-62, 2004. Recuperado de: < https://doi. org/10.1023/B:JSTE.0000031462.40615.56>. Accedido el: 9 oct. 2018.

QUINTANILLA, M. El dilema epistemológico y didáctico del curriculum de la enseñanza de las ciencias: ¿Cómo abordarlo en un enfoque CTS. Revista Pensamiento Educativo, Santiago, v. 25, n. 2, p. 299-334, 1999. Recuperado de: < http://pensamientoeducativo.uc.cl/ index.php/pel/article/view/164/346>. Accedido el: 9 oct. 2018. 
QUINTANILLA, M. Identificación, caracterización y evaluación de competencias científicas en el aula desde una imagen naturalizada de la ciencia. In: QUINTANILLA, M.; ADÚRIZBRAVO, A. (Ed.). Enseñar ciencias en el nuevo milenio: retos y propuestas. Santiago: Ediciones Universidad Católica de Chile, 2006. p. 17-42.

QUINTANILLA, M. Proyecto FONDECYT 1150505: identificación y caracterización de competencias de pensamiento científico (CPC) de educadoras de párvulos en formación inicial docente: una contribución fundamental para la educación científica en las primeras edades. Santiago de Chile: Fondo Nacional de Desarrollo Científico y Tecnológico, 2015.

RAVANAL, E.; QUINTANILLA, M. Concepciones del profesorado de biología en ejercicio sobre el aprendizaje científico escolar. Enseñanza de las Ciencias, Barcelona, v. 30, n. 2, p. 33-54, 2012. Recuperado de: < https://www.raco.cat/index.php/Ensenanza/article/ view/254502>. Accedido el: 9 oct. 2018.

RÍOS, L.; ANGULO, F. Enseñar y aprender ciencias en las primeras edades. In: DAZA, S.; QUINTANILLA-GATICA, M. (Ed.). La enseñanza de las ciencias naturales en las primeras edades: su contribución a la promoción de competencias de pensamiento científico. Barrancabermeja: Litodigital, 2011. v. 5, p. 128-143.

SANMARTÍ, N. Necesidades de formación del profesorado en función de las finalidades de la enseñanza de las ciencias. Revista Pensamiento Educativo, Santiago, v. 30, p. 35-60, 2002.

SANMARTÍ, N.; GARCÍA, P. Interrelaciones entre los enfoques curriculares CTS y los enfoques de la evaluación. Revista Pensamiento Educativo, Santiago, v. 25, p. 265-298, 1999.

SCHLICK, M. Sobre el fundamento del conocimiento. In: AYER, A. J. (Comp.). E1

positivismo lógico. México: Fondo de Cultura Eeconómica, 1965. p. 215-232.

Recepción: 16/03/2018. Aprobación: 08/05/2018.

Contacto: Pontificia Universidad Catolica de Chile, Avenida Vicuña Mackenna, 4860, Santiago 7820436, Chile. 
